\title{
Rotation-Induced Change of Muscle Tone
}

Jürg Kesselring, MD, Department of Neurorehabilitation, Rehabilitation Centre, CH-7317 Valens (Switzerland)

During a circus performance of the fabulous 'Skating Willers' I was chosen by chance to act as a collaborator in their breathtaking rolling show or as I suspect as a pitiable contrast to their unbearable lightness of mastering laws of rotation-induced motor behaviour. The two artists were holding me in between them and were spinning around clockwise on a round table of $2 \mathrm{~m}$ diameter at a speed of $3607 \mathrm{~s}$. Centrifugation forced my body into a horizontal plane, the centre of rotation being approximately around my navel. When put down on to the table after 15 rounds of intense rotation I felt no dizziness but I was convinced that the table had broken down and was tilted towards the right side of my body. With all my strength I intended to prevent the two artists with their roller skates and myself from sloping away. Subsequent video analysis revealed a stable table and unaffected artists but me pushing down to the left side (fig. 1). For at least $10 \mathrm{~s}$ the artists were unable to put me back into the upright position. This phenomenon is known as the 'pusher syndrome' in severely affected hemiplegic patients [1].

The experiment was repeated several times afterwards (without audience) and produced the same effect as it does regularly in several hundreds of public performances with innocent victims. On immediate neurological examination there were slight nystagmus and definitely exaggerated reflexes on both legs, but no extensor plantar responses. There are several lessons to be learnt from this experiment with implications for neurorehabilitation: (1) Clockwise rotation of the body in the horizontal plane (ampullope-tal stimulation of the left and ampullofugal stimulation of the right posterior semicircular canal) provoke no dizziness but the illusion of the ground being tilted to the right side and consequently falling of the body to the left. (2) Spasticity has at least some active

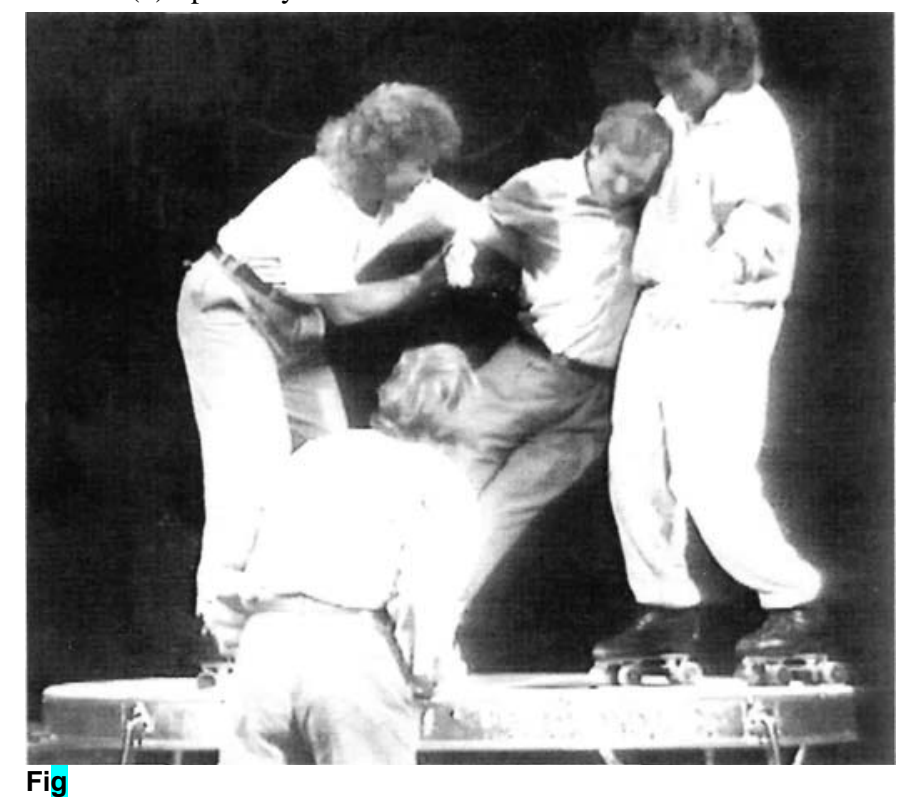

tion.

References

Davies PM: Steps to Follow. A Guide to the Treatment of Adult Hemiplegia. Heidelberg, Springer, 1985.

Kesselring J: Neurology of behaviour as basis for neurorehabilitation. Schweiz Med Wo-chenschr 1992;122:1197-1205. Brandt T, Dieterich M: Pathological eye-head coordination in roll: Tonic ocular tilt reaction in mesencephalic and medullary lesion. Brain 1987;110:649-666.

Gresty MA, Bronstein AM, Brandt T, Dieterich M: Neurology of otolith function. Peripheral and central disorders. Brain 1992; 115: 647-673.

components of searching for information about the relationship between body and environment when perception by other sense organs is disturbed. (3) Neither knowledge about the facts to be expected nor visual guidelines are able to suppress the forces of exaggerated vestibulo spinal reflexes in untrained subjects. (4) Motor behaviour should always be tried to be understood as interaction between organism and environment [2]. The observation supports the hypothesis that vestibulogenic postural asymmetries may be an adjustment of the body long axis to align with what the nervous system erroneously computes as being vertical [3, 4]. 
1994 S. Karger|AG, Basel

0345-0300 $88.00 / 0$ 